\title{
GENOME WATCH
}

\section{True Blood: dengue virus evolution}

\author{
Rachael Wash and Carmen Diaz Soria
}

This month's Genome Watch highlights insights into different selection pressures in dengue virus evolution.

Dengue virus (DENV) infects approximately 400 million people annually. It is a singlestranded RNA virus transmitted by Aedes aegypti or Aedes albopictus mosquitoes in tropical regions of the world. There are four closely related serotypes, DENV-1, DENV-2, DENV-3 and DENV-4. Symptoms of infection include fever, headaches, vomiting, and in severe cases, haemorrhage, shock, organ failure and death. With $\sim 3.6$ billion people at risk, furthering our knowledge of DENV is important, particularly as no vaccines or antiviral drugs are commercially available. Three recent studies now show how genome profiling can advance our understanding of DENV evolution and thus suggest targets for therapeutic strategies.

The DENV genome encodes ten proteins (capsid (C), envelope (E), pre-membrane (prM), nonstructural 1 (NS1), NS2A, NS2B, NS3, NS4A, NS4B and NS5) and has $5^{\prime}$ and 3 ' untranslated regions (UTRs). Genome replication relies on the error-prone viral RNA polymerase (NS5), which generates a population of genetically variable viruses during infection. However, DENV replicates in two hosts (mosquitoes and humans) that produce different selection pressures, which may have an impact on the repertoire of viruses present, influencing how the human immune response or a drug could control infection.

To measure DENV-2 diversity during transmission from human to insect, and how this may shape virus evolution, Sim et al. used whole-genome Illumina sequencing to profile viral RNA that was extracted from patients' plasma or A. aegypti that were fed patients' blood ${ }^{1}$. Levels of intra-host diversity of viral single-nucleotide variants (SNVs; identified with the LoFreq algorithm) were similar in humans and mosquitoes, but only $9.7 \%$ of the 267 SNVs detected in human-derived viruses were also observed in mosquito-derived viruses, suggesting that most mutations occurred at random.

To explore how selection pressures varied in different hosts, the authors compared ratios of non-synonymous to synonymous nucleotide changes in human- and mosquito-derived DENV populations. By looking for regions in the virus genome that were 'hot spots' for SNVs, the authors identified genes with greater diversity and under strong selection pressures in either human-derived (prM, E and NS1) or mosquito-derived (NS3 and $3^{\prime}$ UTR) virus populations. These data suggest that the roles of these genes in virus adaptation may depend on host species.

Using deep sequencing to identify sites under selection also provides the opportunity to identify antiviral targets. Regions of the DENV genome that are conserved provide prospective targets for antivirals, and negative selection may mean that drug-resistant mutations are less probable. Sessions et al. ${ }^{2}$ used Illumina and SOLiD (Sequencing by Oligonucleotide Ligation and Detection) sequencing of DENV-1 isolated from humans or $A$. aegypti and A. albopictus infected with patient sera. They observed sequence variation between isolates from the two mosquito species, suggesting that vector species influence DENV genome diversity. In contrast to Sim et al. ${ }^{1}$, several 'cold spots' (regions in which SNV frequencies are significantly depleted) were detected in NS3 in DENV-1 isolated from mosquitoes, around the ATP-binding domain. Furthermore, RNA interference to target this region significantly reduced viral copy number. These findings led the authors to suggest that NS3 is an antiviral target worthy of future investigation ${ }^{2}$.

Alternatively, antiviral drugs can target host proteins rather than the virus itself, thus reducing the emergence of drug-resistant viral mutations. In a mouse study, Plummer et al. ${ }^{3}$ used Illumina sequencing to investigate the effect of a compound (UV-4B) that targets host $\alpha$-glucosidases on DENV-2 evolution. The authors detected several SNVs that only arose after UV-4B treatment. However, none of the SNVs seemed to enhance virus fitness under the drug regime, as reduction of DENV serum titres by UV-4B was retained, showing its potential for clinical use.

The use of deep sequencing in these studies shows how DENV diversity can be tracked under different selection pressures, which can come from human or invertebrate hosts or drug treatment. This will aid future studies into vaccines and antivirals for DENV and other insect-transmitted viruses.

Rachael Wash and Carmen Diaz Soria are at the Sanger Institute, Wellcome Trust Genome Campus, Hinxton, Cambridge CB10 1SA, UK. e-mail:microbes@sanger.ac.uk doi: $10.1038 /$ nrmicro3570 Published online 6 October 2015

1. Sim, S. et al. Tracking dengue virus intra-host genetic diversity during human-to-mosquito transmission. PLOS Negl. Trop. Dis. 9, e0004052 (2015).

2. Sessions, O. M. et al. Analysis of dengue virus genetic diversity during human and mosquito infection reveals genetic constraints. PLoS Negl. Trop. Dis. 9, e0004044 (2015).

3. Plummer, E. et al. Dengue virus evolution under a hosttargeted antiviral. J. Virol. 89, 5592-5601 (2015).

Competing interests statement

The authors declare no competing interests. 